\title{
Desenvolvimento de aplicação para interfaceamento com experimentos remotos por smarthphones.
}

\author{
Willian Rochadel - UFSC - Universidade Federal de Santa Catarina \\ willian.rochadel@ufsc.br \\ Ellen Larissa de Carvalho Aquino - UFSC - Universidade Federal de Santa Catarina \\ aquino.lari@gmail.com \\ Juarez Bento Silva - UFSC - Universidade Federal de Santa Catarina \\ juarez.silva@ieee.org
}

\begin{abstract}
Resumo
Neste artigo os autores demonstram o desenvolvimento e a importância de uma aplicação adequada para interatividade de experimentos remotos por dispositivos móveis, principalmente smartphones, apresentando o aplicativo do RExLab - UFSC. A popularização destes dispositivos permite o alcance por mais usuários, mesmo pelos estudantes da educação básica. Aliando a pratica de experimentação remota e dispositivos móveis ao conceito de ubiquidade, é demonstrada a criação de aplicações que proporcionem um modo interativo e dinâmico para atrair ainda mais o aluno a utilizar estes experimentos remotos como suporte ao ensino de ciências desde as séries iniciais. Buscando utilizar softwares gratuitos ou de baixo custo, e os recursos de HTML5 e jQuery, é possível implementar páginas compatíveis com diferentes Sistemas Operacionais móveis, como iOS, Android, Windows Phone, alguns Symbian, entre outros. Ainda são apresentados padrões de layouts que colaborem para uma expansível acessibilidade.
\end{abstract}

Palavras-chaves: experimentação remota, educação móvel, open-source, layouts e padrões W3C, HTML5 and jQuery mobile.

\section{Development of application for interfacing with remote experiments by smarthphones.}

\section{Abstract}

In this short paper the authors describe the development and importance of an appropriate application for interactivity of remote experiments by mobile devices, mainly smartphones, presenting the application of RExLab - UFSC. The popularization of these devices allows the scope for more users, even by students of basic education. By combining the concept of remote experimentation and the concept of mobile devices to the concept of ubiquity it is possible to demonstrate the creation of applications that provide an interactive and dynamic way to attract more students to use the remote experiments to support science education from the early grades. The use of free or low cost software, and the resources of HTML5 and jQuery, allows the implementation of pages that are compatible with different mobile operating systems, such as iOS, Android, Windows Phone, some Symbian, among others. Patterns and layouts that collaborate for an expandable accessibility are presented as well.

Keywords: remote experiments, mobile learning, open source, layouts and standards W3C, HTML5 and jQuery mobile. 


\section{Introdução}

A Usabilidade é a capacidade de um determinado produto ser utilizado pelo indivíduo para atingir objetivos específicos com eficiência e satisfação em um contexto exclusivo de interação (Araujo, 2012).

Assim, a relação da mobilidade com acessibilidade torna-se relevante aspecto a ser implementado nos dispositivos de aplicações móveis. Num contexto pedagógico, esta questão concilia em uma interessante ferramenta para solucionar algumas brechas no ensino-aprendizagem.Como nas áreas das tecnologias e das ciências que carecem de realizações de trabalhos experimentais em laboratórios com equipamentos e experiências que demonstrem na prática os conceitos discutidos em sala de aula.

Pois a escassez destes recursos, a falta de laboratórios e mesmo a dificuldade de acesso limita uma aprendizagem mais prática. Logo, se tratando de maneiras de proporcionar uma experiência mais próxima da realidade, por si só já são bastante atrativas aos alunos os simuladores e laboratórios virtuais com experiências simuladas de resultados gravados; Mas, além destes recursos, a utilização de recursos remotos tende a permitir uma experiência ainda mais real (Paladini, 2008).

Ao interagir com experimentos em laboratórios remotos, o usuário tem a experiência de estar trabalhando com equipamentos físicos disponibilizados por pesquisadores de modo a proporcionar esta usabilidade ainda mais agradável. Certamente um desafio na criação de novas experiências, a disponibilização destes recursos deve se preocupar com a própria facilidade proporcionada de operação dos experimentos em qualquer lugar e em qualquer momento, o conceito de ubiquidade (Dochev, 2006).

Logo, proporcionar um envolvimento do aluno com experimentos remotos em seu próprio dispositivo móvel estende ainda mais a utilidade dos laboratórios, pois, afinal, os usuários estão constantemente conectados a estes meios móveis e desta forma podem aprender, revisar e demonstrar os conceitos de ciências mesmo em movimento, no caminho à escola, com amigos ou na própria sala de aula apenas com um aparelho que permita o acesso à internet.

Este conceito amplia o uso de experimentos para além do laboratório ou sala de aula e proporciona que novos usuários tenham o acesso a estes recursos no ônibus, no metrô, em um táxi, ou em filas, onde informações e interfaces adequadas são indispensáveis para atender necessidades imediatas (Krause, 2011).

Há uma expansão da rede de telefonia móvel devido os dispositivos móveis estarem cada vez mais acessíveis e ainda possuírem interessantes recursos tecnológicos que chamam a atenção, tais como, acelerômetro, touchscreen, vibration, location $e$ compass. Poder aliar recursos às ferramentas didáticas proporciona uma interação com o aluno de forma mais atrativa e dinâmica.

Relativamente novo, o Mobile Learning (M-learning) (Sharples, 2003) carece de aplicações que façam proveito de todos os recursos tecnológicos disponíveis num dispositivo móvel, o que ocorre são simples adaptações das páginas ou aplicações de workstations, estas não proporcionam uma experiência inovadora em interação.

A disponibilização destes recursos segue padrões internacionais que a World Wide Web Consortium (W3C ${ }^{1}$ ) recomendam (W3C, 2010) e se baseia em conceitos de Ambientes Virtuais discutidos por Pereira (2007).

\footnotetext{
${ }^{1}$ Disponível em: http://www.w3.org/Mobile/

V. $10 \mathrm{~N}^{\circ} 1$, julho, 2012
} 
Com estes conceitos o Laboratório de Experimentação Remota da Universidade Federal de Santa Catarina tem desenvolvido o REx-Mobile, um aplicativo adaptado para uso de experimentos remotos por diferentes Sistemas Operacionais.

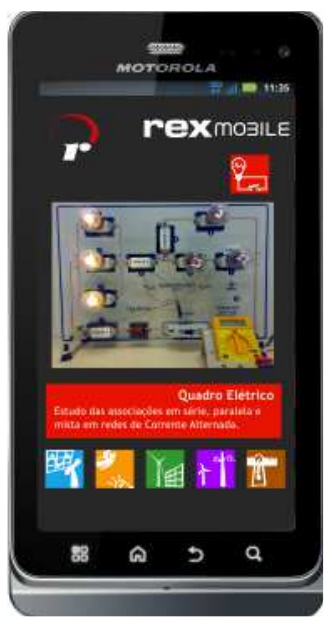

Figura 1. Screenshot do aplicativo RexMobile

Os smartphones possuem a vantagem de serem mais acessíveis, no entanto, carece de alguns recursos, ou até possuem uma qualidade inferior em relação aos dispositivos mais avançados.A experiência de interface e usabilidade que o REx-Mobile busca proporcionar em seu desenvolvimento é uma interatividade simples, adaptável e ainda oferecer um envolvimento de forma agradável aos usuários.

Conceitos aplicados no desenvolvimento da interface, softwares, linguagens e recursos utilizados serão melhor detalhados no decorrer deste artigo.

\section{Necessidade de Ambientes Interativos na Educação}

As Novas Tecnologias da Informação e Comunicação (NTICs) possibilitam a criação de espaços para as interações humanas possibilitando novos processos de aprendizagem, comunicação e construção do conhecimento. Elas têm sido incorporadas à educação em diferentes cenários dando lugar a uma ampla gama de usos. Atualmente é tema corrente de discussão a incorporação relativa aos dispositivos computacionais interconectados, e associa-se diretamente a capacidade das NTICs de criar redes de intercomunicação e interconexão, que por sua vez se relacionam com a criação de novos ambientes de ensino-aprendizagem; ou seja, discute-se, em especial, como a World Wide Web (WWW) pode favorecer o surgimento de novos ambientes de ensinoaprendizagem visto que este cenário proporciona que os alunos possam construir saberes mais além das instituições de ensino. As NTICs possibilitam a aprendizagem à distância demonstrando que já não é as salas de aulas o local exclusivo do saber, e oferecem uma dinâmica que não admitem o monopólio dos conteúdos nem a transmissão destes em um espaço e tempo dados por uma única pessoa possuidora do saber, isto é, o professor.

Com a evolução da Web 1.0, unidirecional, até a Web 3.0 descrita por John Markoff, como a web colaborativa em artigo no New York Times, pode-se pensar que a possibilidade de "criar" ou "produzir" conhecimento é de "todos", gerando um novo paradigma de construção e apropriação do saber que promove um modo não linear, holístico, descentralizado, participativo e colaborativo da aprendizagem, a partir de uma ampla gama de possibilidades de comunicação (redes sociais, wikis, blogs, fóruns, etc.) 
e de produção. Em síntese, dentre os ambientes novos que surgem como resultado da incorporação das NTICs à educação, são destacados especialmente os que se baseiam em configurações construídas sobre a possibilidade de interconexão e intercomunicação que oferecem estas tecnologias, ou seja, os ambientes virtuais ou on-line de ensino e aprendizagem.

Conectados constantemente, novas formas de ensino não são o suficiente para agradar os estudantes, não basta possibilitar acesso ao mesmo recurso que é disponibilizado comumente e inadequados a uma realidade móvel, toda metodologia deve convergir de forma interativa.

No processo educacional, a inclusão dos dispositivos móveis, poderá contribuir para impulsionar estas mudanças metodológicas visando modelos mais atraentes para os usuários digitais, pois, permitem dar continuidade ao processo educativo fazendo uso de dispositivos pequenos, que podem oferecer as mesmas funcionalidades que um computador do tipo desktop ou laptop. Os dispositivos móveis agregam a mobilidade geográfica com a virtual, a qual permite a aprendizagem no momento em que se necessita. A partir disso pode-se entender Mobile learning como uma nova forma de aprendizagem pessoal que nunca termina, um novo modelo tecnológico-pedagógico onde aponta para uma nova dimensão nos moldes de educação, que pode atender as urgências da aprendizagem localizadas em cenários móveis e permitir atividades altamente interativas.

A Mobile learning deve ser entendida como um componente que agrega valor nesse processo de ensino-aprendizagem, uma vez que, proporciona a interconectividade, oferece a liberdade, diminui a dependência de lugar ou espaço e produz a captura de pensamentos e idéias de maneira espontânea, ampliando os limites das salas de aula, permitindo acessar os recursos disponibilizados quando e onde o usuário necessitar, facilita a possibilidade de implementar modos inovadores de ministrar aulas e de aprendizagem.

\section{Incentivos do uso das NTIC's}

A aplicação dos dispositivos móveis estão se tornando cada vez mais acessíveis à maioria das classes sociais, popularizando o acesso aos recursos tecnológicos inovadores e com valores não muito altos.

Incentivos como a isenção de impostos para tablets, anunciado pelo governo, (Nunes, 2011) tem colaborado para este acesso. A redução de preços poderia ser maior segundo o consultor José Martim Juacida, mas "O Brasil sofre com a qualificação dos profissionais. Não há engenheiros em abundância e isso impacta no preço final".

Para universalizar o acesso dos alunos à tecnologia, Fernando Haddad, ministro do MEC, anunciou a distribuição a partir de 2012 de "centenas de milhares" de tablets nas escolas públicas pelo MEC (Dias, 2011). Há notícias de que o edital para aquisição de 300 mil unidades está pronto para ser anunciado (Máximo, 2011).

Os tablets tendem a reduzir rapidamente os preços com os incentivos fiscais e a compra de grandes quantidades pelo governo, assim, classes sociais mais baixas poderão ter acesso ao recurso e usufruir de novas tecnologias.

Não se pode contar com grandes processos nestes dispositivos, logo, o acesso aos ambientes de aprendizagem deve ser facilitado, interagindo com os recursos do touchscreen e algumas opções com o acelerômetro, isto torna o uso mais agradável o que visa atrair a atenção dos estudantes às possibilidades que as novas tecnologias proporcionam.

Estes incentivos de tablets na educação não são apenas no Brasil, o Governo da Tailândia também irá distribuir tablets nas escolas (Morales, 2011). 
Já há tablets feitos com o propósito de utilização na educação, na CES2012 foi apresentado o tablet do projeto One Laptop Per Child (OPLC) (Crothers, 2012), feito para crianças na escola, tem o custo de Us $\$ 100$ e conta com adaptações como a capa de borracha para proteção.

\section{Adequação dos AVA para o ensino-aprendizagem em Dispositivos móveis}

A ubiquidade, principal característica do modelo aplicado ao M-learning, ou seja, uma aprendizagem em qualquer lugar e em qualquer momento (Dochev, 2006), permite estender as barreiras da sala de aula. O emprego de tecnologias específicas é o que diferencia das outras aprendizagens eletrônicas, onde, o dispositivo móvel permite uma aprendizagem enquanto se está em movimento (Bartholo, 2009).

O usuário está em busca de objetividade, no entanto nota-se uma dispersão muito fácil ao utilizar a internet como recurso de educação devido ao uso frequente de redes sociais, mensagens instantâneas, mensagens de e-mail, jogos ou outras diversões, além disso, enquanto utiliza-se um dispositivo móvel, outras tarefas são realizadas simultaneamente, perdendo o foco.

Desta forma, a simples disponibilização de materiais didáticos para dispositivos móveis, não é o bastante, é necessário torná-los adequados a este tipo de ensino, prevendo diferentes distrações e pouco tempo, um recurso interessante é a qualquer momento poder parar os estudos e continuar em outro momento no mesmo ponto que havia ficado pendente, ou então, pular estágios e ter um controle das tarefas pendentes.

Outra preocupação é quanto à configuração dos dispositivos como: sistema operacional, touchscreen, tamanho da tela, capacidade de armazenamento, desempenho, acesso à internet ou recursos de acelerômetro, compass, location e vibration, pois, o usuário pode se frustrar com a limitação de recursos disponibilizados, caso seu aparelho não se adéque a um padrão proposto. Para isso existem padrões para o desenvolvimento como os indicados pela W3C, conforme fig.2.

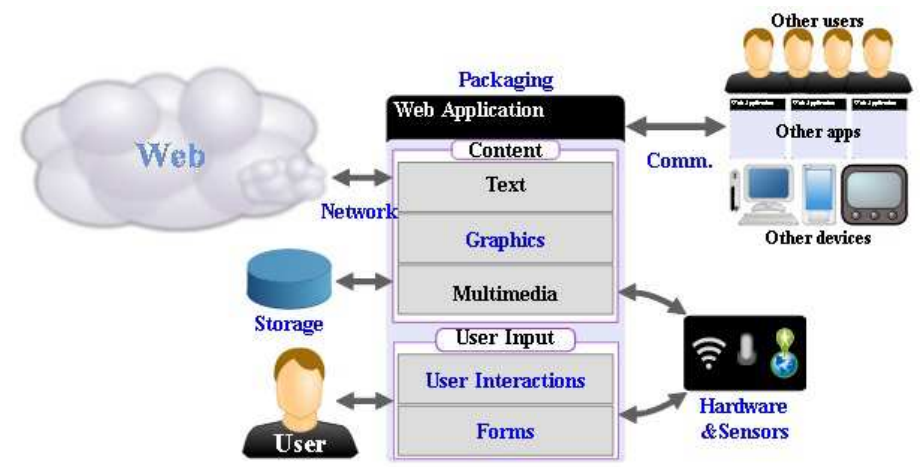

Figura 2. A Web como uma plataforma de desenvolvimento de aplicações (CONNORS, 2012).

Ao permitir esta fácil disponibilidade, é possível alcançar mais usuários e proporcionar uma experiência agradável e não frustrante com seus dispositivos. Deste modo, o interfaceamento com o usuário deve ser facilitado, cumprindo o papel de informar o necessário e, para isto, observa-se os comportamentos da aplicação na projeção da entrada de dados reduzida, devido a limitação dos tamanhos de teclado nos dispositivos.

Com limitações de memória é importante a seleção da informação, para visualizar o que realmente é necessário na pequena tela e reduzir conteúdos longos com a finalidade de não consumir demasiadamente banda larga pelo custo por byte, em 
algumas conexões. As cores também influenciam na afeição pela aplicação, o excesso pode tornar o visual cansativo e pesado.

Para este propósito é importante notar o apoio de novas linguagens que possibilitam integrar funcionalidades interativas e portáveis a outros dispositivos móveis, visto que, ainda há pouco tempo atrás, o desenvolvimento se portava diferentemente para cada plataforma sendo necessária uma adaptação por aparelho.

Por outro lado, outros fatores tem ainda incentivado esta criação com altos investimentos em infraestrutura de rede, maior abrangência de área, redução dos custos, disponibilidade, recursos tecnológicos e boa aceitação do usuário.

\section{Rede de Telefonia móvel}

A visualização dos experimentos necessita de um bom acesso à internet devido ao streaming de vídeo, este acesso pode ser provido pelo uso da terceira geração de tecnologias e padrões de telefonia móvel, com velocidades comparadas à Banda Larga e que possibilita uma boa velocidade de conexão.

Há uma rápida expansão do acesso à rede móvel 3G e investimentos altos para a infraestrutura da rede 4G no Brasil, motivados pela Copa 2014 e Olimpíadas de 2016. Serão Us $\$ 5,4$ bilhões gastos para licitações e infraestrutura, que inclui estender um cabo de fibra óptica submarino até os Estados Unidos, com esta expansão as empresas pretendem oferecer pacotes de serviços. Diversos pontos de internet grátis também estão sendo implantados para que as pessoas de baixa renda possam acessar a teia global, de modo a popularizar o acesso à informação.

A idéia é que, expandindo o local de estudo e a interatividade com os dispositivos, o aluno possa rever um assunto antes da aula ou interagir com os experimentos em diferentes locais, talvez compartilhando com outros colegas e família demonstrando as experiências e o conhecimento adquirido.

\section{Material de Ensino}

Pereira (2007) descreve Ambiente Virtual de Aprendizagem (AVA) como uma opção de mídia utilizada para mediar o processo ensino-aprendizagem a distância. $\mathrm{O}$ RExLab utiliza o Modular Object-Oriented Dynamic Learning Environment (Moodle²), um AVA open source e gratuito que permite o desenvolvimento de sites web dinâmicos, como também, partilhar conteúdos didáticos, recursos como chat, fórum e atividades personalizadas em diferentes modos de questionários. Estas possibilidades disponibilizadas pelo Moodle facilitam a produção e distribuição dos materiais de ensino, os recursos integrados à um banco de dados MySQL ainda permite a gestão de aprendizagem, avaliação dos alunos, controle de acesso e suporte pedagógico.

O plugin Mobile Learning Engine do Moodle (MLE-Moodle ${ }^{2}$ ) é um módulo também open source, oferecido gratuitamente e customizável que permite acesso aos recursos do Moodle, sendo instalado no mesmo servidor e sem a necessidade de instalar nenhum recurso adicional no dispositivo móvel.

Este plugin provê a camada de adaptação que interage com a proposta de Pereira (2007) nas situações:

- Documentação e Informação: download e upload de arquivos, oferecendo suporte no uso do AVA e apresentação adaptada do material didático, do conteúdo e das informações institucionais.

- Comunicação: adaptação das informações de forma síncrona e assíncrona em relação à necessidade do dispositivo.

\footnotetext{
${ }^{2}$ Disponível em http://mle.sourceforge.net/

V. $10 \mathrm{~N}^{\circ} 1$, julho, 2012
} 
- Gerenciamento pedagógico e administrativo: perfis diferentes para acesso e visualização do desempenho, das avaliações ou consultas, dependendo do tipo do usuário.

- Produção: desenvolvimento e resolução das atividades ou problemas dentro do ambiente.

A vasta gama de configurações e a diversificação dos recursos disponibilizados, necessitam de adaptações específicas, nesta linha, a implementação do portal mobile do RExlab com o MLE-Moodle possibilita que usuários com diferentes dispositivos móveis tenham acesso à interface WEB sem a restrição do sistema operacional, isto lhe permite acessar os experimentos, realizar seus estudos e completar suas atividades, sem a exigência de poderosos aparelhos.

O material didático que pode ser baixado do RExLab em dispositivos móveis são em Portable Document Format (.pdf) e as apresentações em PowerPoint (.ppt), formatos que a maioria dos dispositivos móveis pode visualizar e se acomodam aos diferentes formatos de tela. Estes conteúdos são simples para não tornar a leitura enfadada, destacando os pontos mais importantes e oferecendo exemplos.

Outro interessante recurso que pode facilitar o compartilhamento e o acesso a estes conteúdos online é o QR-code, um código de barras em $2 \mathrm{D}$ que, quando escaneado, é convertido para um link.

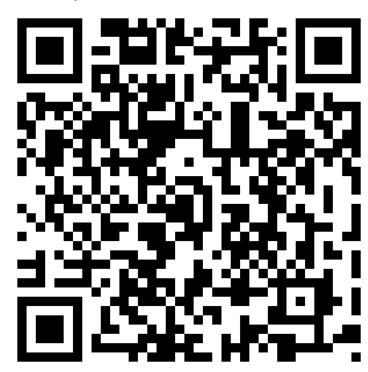

Figura 3. QR-Code for access at RExLab portal.

Outra preocupação quanto ao material didático disponibilizado é a objetividade, pois, o usuário não costuma ficar longos períodos com o dispositivo nas mãos e a concentração neste momento é dividida com outras tantas tarefas. Dentro da produção para experimentação remota, é fundamental organizar um modelo mental de dados, funções e atividades específicas, afim do aluno compreender e reconhecer estes elementos.

\section{Experimentos Remotos Interativos}

Os experimentos remotos são elementos físicos que interagem por comandos ativados remotamentente através da Web em experimentos ligados a microservidores Web ou placas Arduino com porta ethernet (Silva, 2007). Desta forma, as interações são diretas com equipamentos reais controlando relés, circuitos ou sensores, e se obtém o feedback em tempo real dos resultados das experiências online, observado-os através de streaming de vídeo de uma câmera IP direcionada.

Como importante atrativo tecnológico ainda, a interface adaptada ao touchscreen proporciona um melhor controle e maior realidade, mesmo mediada à distância. Os mais simples dispositivos já contam com este recurso, sendo facilmente adaptados às interfaces que utilizam o mouse para interagir.

Uma aplicação prática e interativa, com layout adequado atrai a atenção para o estudo e, neste caso, relacionando com experimentos reais, tende a aumentar o interesse por toda a tecnologia envolvida, tendo controle destes fenômenos vistos até então apenas na teoria. E ainda, permitem a demonstração de mais conceitos estudados nas 
aulas de ciência em sala e as vantagens práticas de entendê-los, beneficiando a aprendizagem dos alunos.

\section{Desenvolvimento do Aplicativo para a Metodologia Proposta}

A partir destes tópicos apresentados e da importância em permitir o fácil acesso ao maior número de usuários em qualquer lugar e a qualquer tempo, a criação do REx-Mobile para interação de experimentos em laboratórios remotos, percebe-se a necessidade de integrar linguagens e tecnologias que incentivem o uso.

Neste sentido, é notório o avanço e desenvolvimento na linguagem HTML5 (Hypertext Markup Language, versão 5), que permite usufruir de grandes recursos dos dispositivos, aprofundar a imersão do usuário, garantindo fácil portabilidade a diferentes dispositivos. Este desenvolvimento em rápida expansão com intenso incentivo tem permitido o desenvolvimento de novas ferramentas atrativas ao uso.

Outro recurso interessante é a definição de estilos com CSS3 (Cascading Style Sheets, versão 3) para as páginas desenvolvidas, com esta linguagem variante do XML (Extensible Markup Language) é possível criar padrões leves e de boa aparência.

Estes interessantes recursos de desenvolvimento são integrados pelo framework jQuery Mobile $^{3}$, que emprega um sistema unificado apropriado para aplicações Web em aparalhos móveis (Silva, 2012) e com utilização de JavaScript de alto nível, gerando códigos compatível com iOS, Android, Windows Phone, Symbian, BlackBerry, entre outros importantes Sistemas Operacionais móveis.

Uma criação ainda facilitada pelos recursos da ferramenta ThemeRoller que permite a definição destes padrões online ${ }^{4}$. Ferramentas que garantiram padrões úteis para o desenvolvimento da aplicação.

Além destes recursos, utiliza-se um código JavaScript que submete comandos CGI (Common Gateway Interface) ao microservidor acoplado ao experimento, acionando-o e permitindo a visualização dos efeitos através de um streaming de vídeo em JPEG, também suportável em diferentes plataformas móveis.

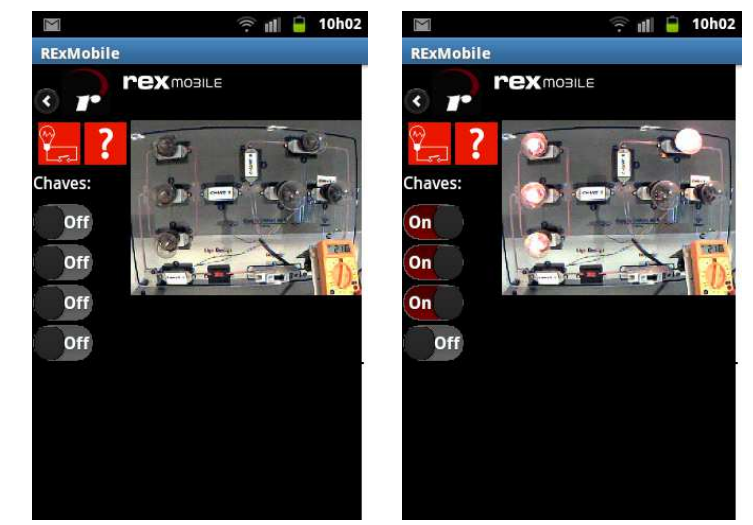

Figura 4. Interação do experimento remoto com o aplicativo em um Samsung S830i com SO Android 2.3

Por fim, o aplicativo permite o acesso às atividades e o material didático disponibilizado no Moodle. Desta forma, o aluno aprende a teoria com o conteúdo específico para o estudo dos efeitos físicos relacionados ao experimento, interage e visualiza os efeitos que ocorreram com este e ainda realiza suas atividades através da mobilidade.

\footnotetext{
${ }^{3}$ Disponível em http://jquerymobile.com/

${ }^{4}$ Disponível em http://jquerymobile.com/themeroller/

V. $10 \mathrm{~N}^{\mathrm{o}}$ 1, julho, 2012
} 


\section{Conclusões}

Neste modelo apresentado, pretende-se contextualizar a vivência e a aprendizagem, tornar o estudo mais atrativo, focando o ensino na aplicação, e ao mesmo tempo exigindo constante acesso que ampliam o campo de atuação e proveito da disciplina além das barreiras da sala de aula, diferenciando do simples uso de laboratórios virtuais que apenas simulam as experiências e não interagem com equipamentos reais, onde os resultados ou manipulação das experiências não reproduzem fielmente a realidade.

A vantagem do uso de Laboratórios Remotos focado no ambiente educacional, com alvo no desenvolvimento de experimentos, adicionada a participação dos professores e colaboração dos alunos, permite projetar utilidades com melhor produtividade de ensino, tornando-o mais eficiente e aplicado ao cotidiano, como forma de abranger ainda mais as necessidades. A partir do dispositivo com acesso a internet, o aluno pode acessar em qualquer momento as experiências disponíveis nos laboratórios, interagir com os equipamentos reais e conferir os conceitos estudados em aula na prática, para relacionar o conhecimento com a observação dos experimentos.

Esta relação dinamiza, assim, o uso da internet nas escolas, não apenas como um recurso sub-utilizado de pesquisa, mas como ferramenta interdisciplinar proveitosa ao ensino-aprendizagem e desenvolvimento de conceitos próprios. Também incentiva os alunos a seguirem no estudo das áreas científico-tecnológicas por aplicarem as atividades que relacionam os conceitos teóricos à prática e interligando a usabilidade destas áreas da ciência. Segundo Antunes (2002):

"[...]aprender contribui para o crescimento pessoal porque quem verdadeiramente aprende não copia ou reproduz a realidade, antes aprende quando é capaz de elaborar uma representação pessoal sobre um objeto da realidade ou conteúdo que busca conhecer."

Por tanto, os Ambientes virtuais de aprendizagem e a experimentação remota se apresentam como ferramentas idôneas para o processo de ensino-aprendizagem colaborativo, a abrangência com o acesso a partir de dispositivos móveis permite ainda mais oportunidades atraentes e interativas para este ensino. Elas oferecem uma interessante perspectiva para o ensino social e colaborativo em múltiplas e distribuídas aplicações. Estas novas tecnologias podem promover um elevado grau de imersão, proporcionando uma sensação de presença real e comunicação em qualquer lugar.

Logo, a vantagem do uso de Laboratórios pelo Mobile learning, destina o uso dos aparelhos a uma melhor produtividade no ensino, designando de forma mais eficiente e aplicado ao cotidiano, o que abrange ainda mais as necessidades. Assim, o aluno pode acessar em qualquer momento as experiências disponíveis nos laboratórios e compor os conceitos estudados em sala de aula, associando o conhecimento com a observação dos experimentos.

A motivação deste projeto está em desenvolver metodologias eficientes e atrativas através de ambientes virtuais de aprendizagem e experimentação remota, adaptados a estes dispositivos móveis. A aplicação através da pesquisa, desenvolvimento e implementação permite prover uma arquitetura educacional baseada no uso de NTICs como suporte para estas soluções com baixo custo, levando em consideração os aspectos cognitivos e pedagógicos do processo de ensino aprendizagem. 


\section{Referências Bibliográficas}

ANTUNES, C. Novas Maneiras de Ensinar. Novas Maneiras de Aprender. Porto Alegre: Artmed, 2002. 172 p.

ARAUJO, E.C.J. et al. Evaluate the usability of mobile devices, João Pessoa, Mar. 2012. Scientific Journal of the Institute for Specialized Education of Paraíba. Disponível em: <http://www.fatecjp.com.br/revista/artigo06.pdf.> Acessado em: Janeiro de 2012

BARTHOLO, V.F.; AMARAL, M. A.; CAGNIN, M.I.Uma Contribuição para a Adaptalidade de Ambientes Virtuais de Aprendizagem para Dispositivos Móveis. Revista Brasileira de Informática na Educação. V 17, n2, 2009.

CONNORS, A.;SUlliVAN, B. Mobile Web Application Best Practices. Dec. 2010. W3C. Disponível em: <http://www.w3.org/TR/2010/REC-mwabp-20101214/> Acessado em: Janeiro de 2012

CROTHERS, B. \$100 OLPC tablet to debut at CES. Jan. 2012. Disponível em:<http://ces.cnet.com/8301-33363_1-57354391/\$100olpc-tablet-to-debut-at-ces/> Acessado em: Abril de 2012.

DIAS, A. MEC will distribute tablets to public school students in 2012. jul.2011. Espaço Educador. Disponível em:<http://espacoeducadoremacao.blogspot.com/2011/09/mec-vai-distribuir-tablets-para-alunos.html >Acessado em Abril de 2012

DOCHEY, D.; HRISTOY, I. Mobile Learning Applications Ubiquitous Characteristics and Technological Solutions. Bulgarian Academy Of Sciences Cybernetics And Information Technologies, Bulgaria, vol.6, no 3, Sofia, 2006.

KRAUSE, M.C.;MOZZAQUATRO, P.M.; Object of mobile learning adapted defaults of usability and accessibility. Rio Grande do Sul, 2011.Exhibit II Scientific Initiation of Computer Science. Disponível em:<http://www.ctec.unicruz.edu.br/labins/JAC2011/artigos/arsha_objeto_de_aprendizagem_movel_adaptado_a_padroes_de_usabi lidade_e_acessibilidade.pdf>Acessado em: Fevereiro de 2012.

MAXIMO, L. Escola pública do país entra na onda dos tablets. São Paulo, dez. 2011. Valor. Disponível em: <http://www.valor.com.br/impresso/brasil/escola-publica-do-pais-entra-na-onda-dos-tablets> Acessado em: Março de 2012.

MOODLE. Sobre. Disponível em: < http://moodle.org/about/>. Acesso em: Fevereiro de 2012.

MORALES, F.Governo tailandês distribuirá tablets em escolas. Nov. 2011. Geek. Disponível em:<http://henrique.geek.com.br/posts/18279-governo-tailandes-distribuira-tablets-em-escolas> Acessado em: Março de 2012.

NUNES, V.K.Isenção de impostos para tablet pode estimular queda de até $15 \%$ nos preços. São Paulo, out. de 2011.Info Money. Disponível em: <http://www.infomoney.com.br/tecnologia/noticia/2232681isencao+impostos+para+tablet+pode+estimular+queda+ate+nos+precos> Acessado em: Março de 2012

PAGANI, T.Usabilidade de interfaces para dispositivos móveis: o que muda nas questões de usabilidade quando estamos falando em dispositivos móveis?Nov. 2011. Tableless. Disponível em:<http://tableless.com.br/usabilidade-de-interfaces-para-dispositivosmoveis-parte1/> Acessado em: Abril de 2012

PALADINI, S.J.B.;SILVA, J.B.;ALVES, G.R.;FISCHER, B.T.;ALVES, J.B.Using Remote Lab Networks to Provide Support to Public Secondary School Education Level. In:IEEE International Conference on CSEWORKSHOPS '08. Computational Science and Engineering Workshops. 11, 2008, São Paulo.

PEREIRA, A.T.C.; SCHMITT, V.A.C. Ambientes Virtuais de Aprendizagem. Pereira, A.T.C. (Org), AVA -Ambientes Virtuais de Aprendizagem em diferentes contextos.Rio de Janeiro: Editora Ciência Moderna. 2007. Cap.1, p. 4-22.

SHARPLES, M. Disruptive Devices: Mobile Technology for Conversational Learning. International Journal of Continuing Engineering Education and Lifelong Learning. Birmingham, v12, 5/6, pp. 504-520, 2003.

SILVA, J. B. A Utilização de Experimentação Remota como Suporte para ambientes colaborativos de aprendizagem. Florianópolis: UFSC, 2007. Tese de Mestrado.

SILVA, M.S. jQuery Móbile - Desenvolva aplicações web para dispositivos móveis com HTML 5, CSS\#, AJAX, jQuery e jQuery UI. São Paulo: Novatec, 2012. 